\title{
A Framework for Diagnosis of Covid-19 Infection using Deep Learning Approach
}

\author{
J. Palimote ${ }^{1}$, E. Osuigbo ${ }^{2}$ L. Atu ${ }^{3}$ \\ Department of Computer Science, Kenule Beeson Saro-wiwa Polytechnic, Bori River State, Nigeria ${ }^{1,2,3}$
}

\begin{abstract}
Coronavirus is a respiratory sickness that is impelled by a novel Covid. The basic indications show up in the contaminated individual are fever, cough, sore throat, and trouble in relaxing. Disappearing of taste, sluggishness, throbs, and nasal blockage can likewise be seen in certain patients. The length among tainting and the principal sign of manifestations might be reached out to 14 days [4]. The disease of this infection is communicated through the beads of patients, for example, coughing and sniffling. In the event that the individual comes by implication or in a roundabout way contact with a contaminated individual, at that point the reached individual gets tainted. The antibodies/medications of this sickness are not accessible as of not long ago. Segregation and social distancing are the lone answers for this disease. Hence, the early recognition of tainted people is needed to stop the spread of contamination. This paper presents a framework for diagnosis of covid-19 infection using Deep Learning approach. The proposed system starts by making use of a covid-19 dataset, which is made up of 6 columns and 48 rows. The dataset comprises of most covid-19 symptoms ranging from dry cough, sore throat, high fever and difficulty in breathing of 48 patients and also the results which shows if the patients is infected with covid-19 or not. We made use of a feed forward neural network in training our model and we had an accuracy of about $92 \%$. The trained model was saved and deployed to web using python flask so that users can enter in most covid-19 symptoms and check if they have been tested positive to the virus or not.
\end{abstract}

Keywords: Deep Learning, Covid-19, Feed Forward Neural Network, Diagnosis System

\section{INTRODUCTION}

The COVID-19 pandemic, brought about by the SARS-CoV-2 infection, has prompted in excess of eighty million affirmed cases, with over 1.76 million passing's universally, as of December 27, 2020. The living and working states of billions of individuals worldwide have been altogether disturbed because of various types of social distancing and lockdowns in numerous urban communities. The world economy has been amazingly debilitated because of business closures and significant limitations on movement. Far reaching accessibility of exact and quick testing systems is amazingly significant in unwinding the perplexing elements engaged with SARS-CoV-2 disease and insusceptibility. To this end, research facilities, colleges, and organizations around the globe have been dashing to create and deliver basically required test kits [1].

Early side effects in COVID-19 patients incorporate fever, dry cough, windedness, cerebral pain, muscle irritation, and exhaustion [2] However, the manifestations are not deterministic because of the recognizable proof of asymptomatic SARS-CoV-2 transporters and the covering highlights with other intense respiratory viral diseases, for example, flu [3]. Hence, profoundly touchy and explicit symptomatic strategies that can recognize COVID-19 cases from solid or other infection tainted people are fundamental for illness the board and therapeutics. Right now, different associations have revealed an assortment of strategies for the clinical determination of COVID-19, which have various standards, tasks, expenses, and sensitivities.

Coronavirus is a respiratory sickness that is impelled by a novel Covid. The basic indications show up in the contaminated individual are fever, cough, sore throat, and trouble in relaxing. Disappearing of taste, sluggishness, throbs, and nasal blockage can likewise be seen in certain patients. The length among tainting and the principal sign of manifestations might be reached out to 14 days [4]. The disease of this infection is communicated through the beads of patients, for example, coughing and sniffling. In the event that the individual comes by implication or in a roundabout way contact with a contaminated individual, at that point the reached individual gets tainted. The antibodies/medications of this sickness are not accessible as of not long ago. Segregation and social distancing are the lone answers for this disease. Hence, the early recognition of tainted people is needed to stop the spread of contamination.

The most generally utilized COVID-19 discovery strategy is constant polymerase chain response (RT-PCR). Notwithstanding, RT-PCR units are expensive and require 6-9 hours to affirm disease in the patient [5]. Because of less affectability of RT-PCR, it gives high bogus negative outcomes. To determine this issue, radiological imaging strategies, 


\section{International Journal of Advanced Research in Computer and Communication Engineering}

Vol. 10, Issue 2, February 2021

DOI 10.17148/IJARCCE.2021.10213

for example, chest X-rays and figured tomography (CT) are utilized to recognize and analyze COVID-19. Coronavirus uncovers some radiological marks that can be handily identified through chest X-rays. For this, radiologists are needed to break down these marks. Notwithstanding, it is a tedious and blunder inclined errand. Consequently, there is a need to mechanize the examination of chest X-rays.

\section{RELATED WORKS}

Srivastava et.al, [6] present a diagram of the COVID-19 pandemic and nanomaterials-empowered bio-sensing approaches that have been as of late detailed for the analysis of SARSCoV-2. In spite of the fact that restricted examinations on the advancement of nanomaterials empowered bio-sensing procedures for the analysis of SARS-CoV-2 have been accounted for, their review sums up nanomaterials intervened improved bio-sensing techniques and the potential instruments that might be answerable for the conclusion of the COVID-19 illness. It is investigated that nanomaterials for example gold nanostructures, lanthanide-doped polysterene nanoparticles (NPs), graphene and iron oxide NPs can be possibly used to create progressed strategies offered by colorimetric, amperometric, impedimetric, fluorescence, and optomagnetic based bio-sensing of SARS-CoV-2. At long last, basic issues that are probably going to quicken the improvement of nanomaterials-empowered bio-sensing for SARS-CoV-2 contamination. Their survey may fill in as a guide for the improvement of cutting edge methods for nanomaterials empowered bio-sensing to satisfy the current interest of ease, fast and early analysis of COVID-19 contamination.

Albahri et.al, [7] proposes a definite approach for the assessment and benchmarking of Artificial Intelligence procedures utilized in all order errands of COVID-19 clinical pictures as future headings; such strategy is introduced based on three successive stages. Right off the bat, the identification methodology for the development of four choice grids, specifically, twofold, multi-class, multi-named and various leveled, is introduced based on the crossing point of assessment rules of every arrangement assignment and AI grouping strategies. Also, the advancement of the MCDA approach for benchmarking AI grouping procedures is given based on the incorporated insightful chain of importance cycle and VlseKriterijumska Optimizacija I Kompromisno Resenje strategies. Finally, level headed and abstract approval methods are depicted to approve the proposed benchmarking solutions.

Das et.al, [8] chooses chest X-rays over CT scan images. For the recognition of Coronavirus. The purpose for this is that $\mathrm{X}$-rays machines are accessible in the vast majority of the emergency clinics. X-rays machines are less expensive than the CT filter machine. Other than this, X-beams has low ionizing radiations than CT images. Coronavirus uncovers some radiological marks that can be handily recognized through chest X-rays. For this, radiologists are needed to dissect these marks. Notwithstanding, it is a tedious and blunder inclined errand. Subsequently, there is a need to computerize the examination of chest X-rays. The programmed examination of chest X-rays should be possible through machine learningbased methodologies, which may quicken the investigation time. These methodologies can prepare the loads of organizations on enormous datasets just as tweaking the loads of pre-prepared organizations on little datasets. Nonetheless, these methodologies applied to chest X-rays are exceptionally restricted. Thus, the primary goal of this paper is to build up a mechanized machine learning-based methodology for identification of COVID-19 contamination in chest X-rays by utilizing the outrageous adaptation of the Inception (Xception) model.

Carter et.al, [1] portrays different examine methods and tests for COVID-19 finding. Most tests for early recognition of SARS-CoV-2 RNA depend on the opposite record polymerase chain response, however isothermal nucleic corrosive intensification measures, including record interceded enhancement and CRISPR-based systems, are promising other options. Distinguishing proof of people who have created antibodies to the SARS-CoV-2 infection requires serological tests, including compound connected immunosorbent measure (ELISA) and horizontal stream immunoassay. This research additionally gives a review of current advancement in COVID-19 indicative methods and items to encourage future improvement and development.

Yuan et.al, [9] gives an exhaustive rundown and correlation of various SARS-CoV-2 analytic techniques for specialists and clinicians to create proper methodologies for the convenient and viable identification of SARS-CoV-2. The overview of current biosensors and demonstrative gadgets for viral nucleic acids, proteins, and particles and chest tomography will give understanding into the advancement of novel point of view strategies for the determination of COVID-19.

Chau et.al, [10] examines the mechanical advances in testing capacity and strategy that are right now utilized or being developed for identifying the novel Covid. They portray the current clinical diagnostics and innovation, including atomic and serological testing draws near, for serious intense respiratory syndrome-coronavirus 2 (SARS-CoV-2) testing just as address their preferences and restrictions. Nucleic corrosive application innovation for atomic diagnostics remains the highest quality level for infection recognition. They feature elective atomic discovery strategies utilized for creating novel COVID-19 diagnostics not too far off. Immunizer reaction against SARS-CoV-2 remains inadequately comprehended 
and legitimate approval of serology tests is important to show their precision and clinical utility. To manage the pandemic, we should accelerate the advancement of quick and far and wide testing through upgrades in clinical diagnostics and testing innovation just as admittance to these devices.

\section{METHODOLOGY}

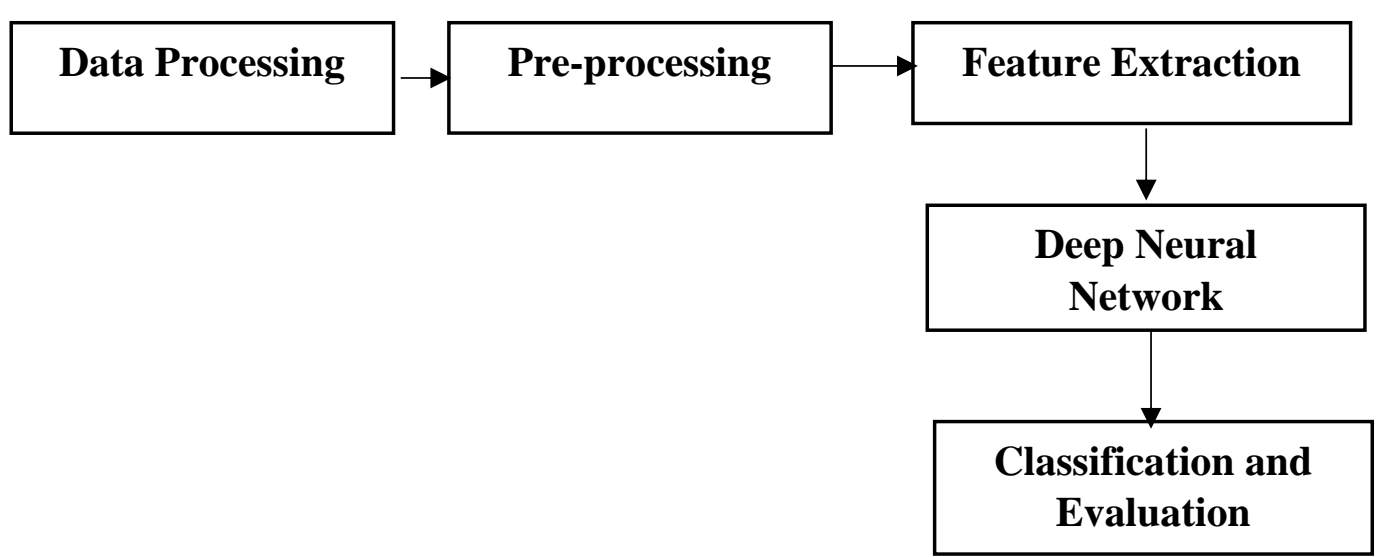

Figure 1: Architecture of the proposed system design

Data Collection: The proposed system uses a covid-19 dataset, which was downloaded from kaggle.com. The dataset is made of six columns and 48 rows. The columns consist of covid-19 symptoms like Dry Cough, Sore Throat, High Fever, Difficulty in Breathing, and Infected with Covid-19.

Pre-Processing: The dataset was preprocessed by removing some NAN values, infinite values and the conversion of words to 0 s and $1 \mathrm{~s}$ for easy fitting of the deep learning algorithm.

Feature Extraction: This has to do with the removal of unwanted columns/ features from the dataset. Therefore, reducing the dimension of the dataset to a pre-defined function. We renamed all the columns from Dry Cough: dry_cough, Sore Throat: sore_throat, High Fever: high_fever, Difficulty in Breathing: difficult_breath, Infected with Covid-19: covid19_result.

\begin{tabular}{rrrrrr} 
& Dry Cough & High Fever & Sore Throat & Difficulty in breathing & Infected with Covid19 \\
\hline 0 & 0 & 2 & 3 & 0 & No \\
1 & 15 & 15 & 20 & 16 & Yes \\
2 & 4 & 5 & 0 & 0 & No \\
3 & 4 & 7 & 9 & 10 & No \\
4 & 0 & 0 & 1 & 0 & No
\end{tabular}

Figure 2: Features Columns of the covid-19 dataset

Deep Neural Network: We made used of a Feed Forward Neural network with a total of three layers which comprises of an input layer, 1 hidden layer and an output layer which output the result to be either 0 (tested negative for covid-19) or 1 (tested positive for covid-19).

Classification and Evaluation: After successful training and scoring of the deep learning model, the trained model will be saved and deployed to web using python flask for evaluation/ testing of the models performance.

\section{DISCUSSION AND RESULTS}

We propose a framework for diagnosis of covid-19 using a deep learning approach. The proposed system starts by making use of a covid-19 dataset, which is made up of 6 columns and 48 rows. The dataset comprises of most covid-19 symptoms ranging from dry cough, sore throat, high fever and difficulty in breathing of 48 patients and also the results which shows if the patients is infected with covid-19 or not. The dataset was preprocessed by removing for NAN values, Infinite Values, and Converting of words to $0 \mathrm{~s}$ and $1 \mathrm{~s}$. Feature extraction is being used in removing unwanted features and 


\section{International Journal of Advanced Research in Computer and Communication Engineering}

Vol. 10, Issue 2, February 2021

\section{DOI 10.17148/IJARCCE.2021.10213}

reducing the dimension of the dataset. The dataset was split into a training set and a testing set. The training set contains $70 \%$ of the dataset while the testing set contains $30 \%$ of the dataset. The training set was used as input to the feed forward neural network with a total input neurons of 48, 64 dense layer (32 each), training epoch $=150$ and a batch size $=50$. The trained model had an accuracy of $92 \%$ which can be seen in figure 5. Figure 7 and 8 shows the graphical interface for diagnosis of covid-19, where users will input the level of different covid-19 symptoms in other to check if they are being infected with covid-19 or not.

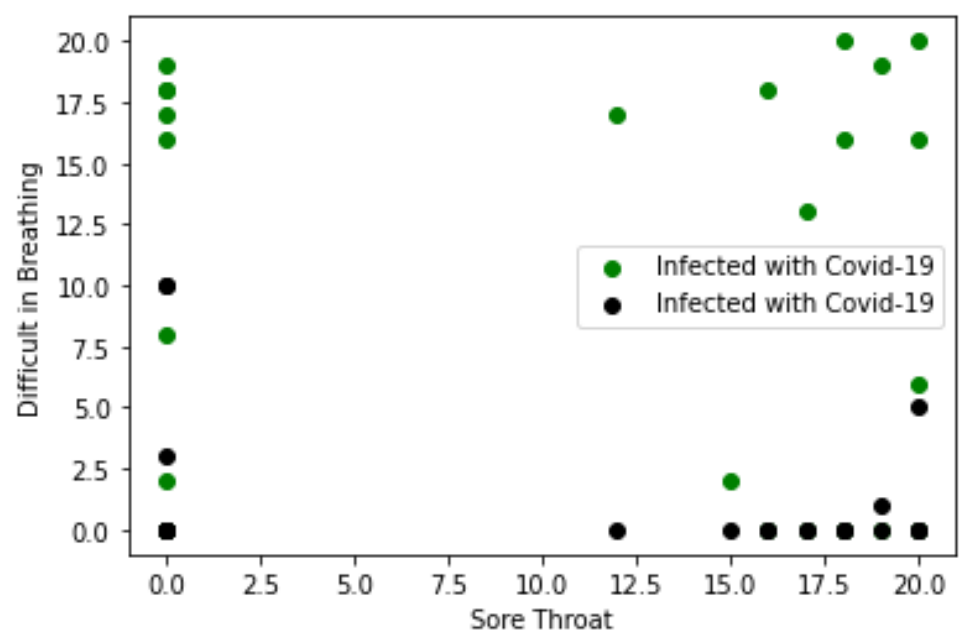

Figure 3: This shows a scatter plot of the dataset. The green dots indicate people infected with covid-19 while the black dots indicated people with negative result of covid-19.
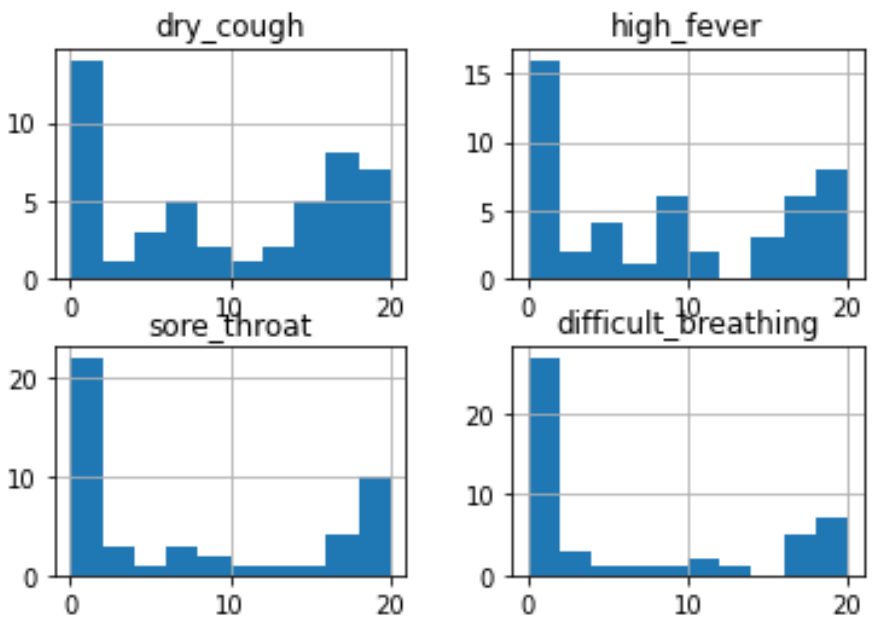

Figure 4: Histogram of various covid-19 symptoms

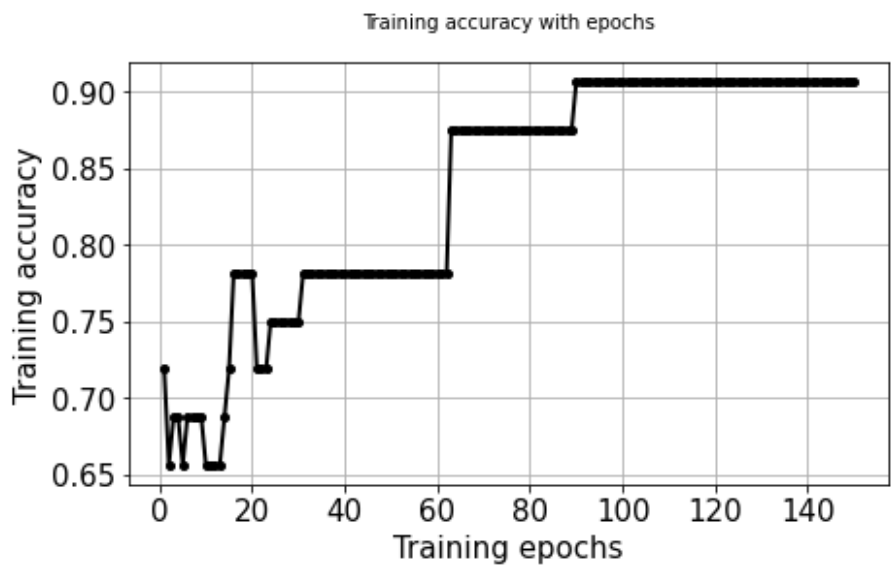

Figure 5: Training Accuracy, our proposed model had an accuracy of about $92.3 \%$ 
International Journal of Advanced Research in Computer and Communication Engineering

Vol. 10, Issue 2, February 2021

DOI 10.17148/IJARCCE.2021.10213

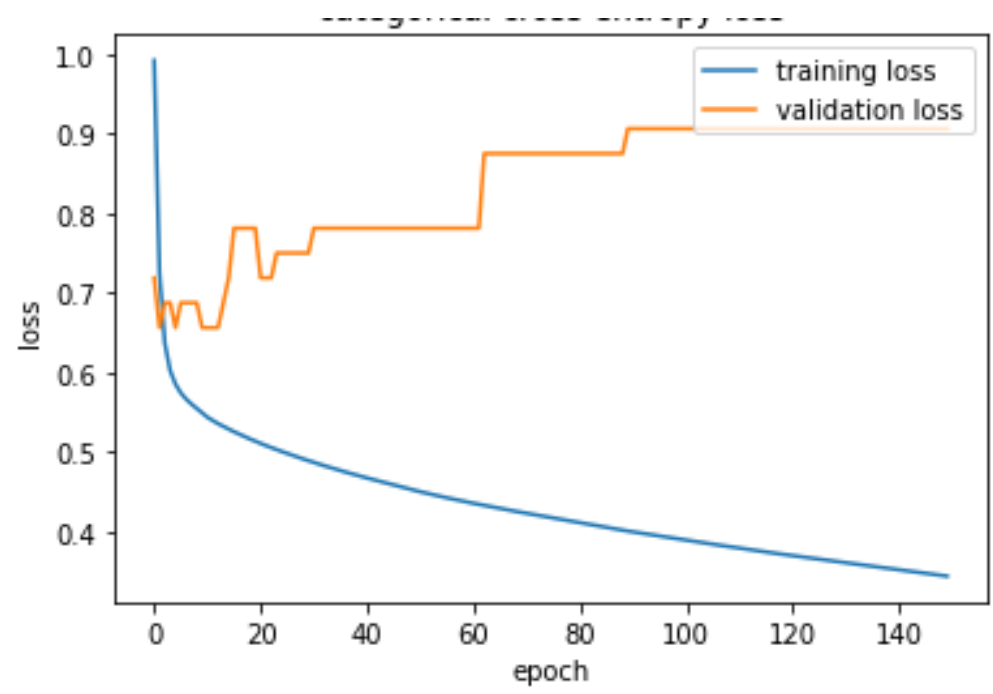

Figure 6: Training loss vs validation loss

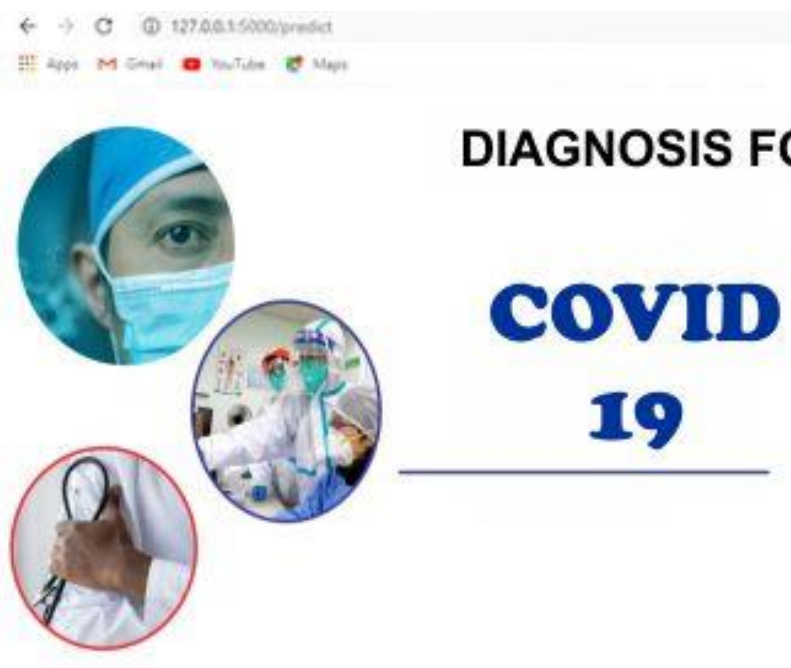

\section{Result}

Figure 7: Diagnosis system which shows that a patient has been infected with covid-19 after entering various covid-19 symptoms 


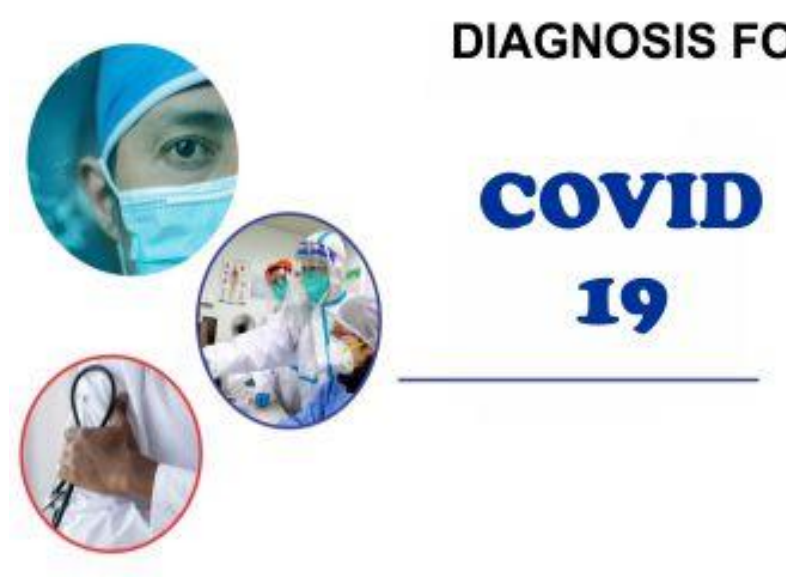

\section{Result}

\section{Your are infected with covid-19. please stavisolated and reoust for a doctor}

Figure 8: Diagnosis system which shows that a patient is being tested negative with covid-19 after entering various covid-19 symptoms.

\section{CONCLUSION}

The COVID-19 pandemic, brought about by the SARS-CoV-2 infection, has prompted in excess of eighty million affirmed cases, with over 1.76 million passing's universally, as of December 27, 2020. The living and working states of billions of individuals worldwide have been altogether disturbed because of various types of social distancing and lockdowns in numerous urban communities. The world economy has been amazingly debilitated because of business closures and significant limitations on movement. This paper presents a diagnosis system for covid-19 detection using deep learning approach. The proposed system starts by making use of a covid-19 dataset, which is made up of 6 columns and 48 rows. The dataset comprises of most covid-19 symptoms ranging from dry cough, sore throat, high fever and difficulty in breathing of 48 patients and also the results which shows if the patients is infected with covid-19 or not. We made use of a feed forward neural network in training our model and we had an accuracy of about $92 \%$. The trained model was saved and deployed to web using python flask so that users can enter in most covid-19 symptoms and check if they have been tested positive to the virus or not.

\section{REFERENCES}

[1]. L. J. Carter, L. V. Garner, J. W. Smoot, Y. Li, Q. Zhou, C. J. Saveson, J. M. Sasso, A. C. Gregg, D. J. Soares, T. R. Beskid, S. R. Jervey, C. Liu "Assay Techniques and Test Development for COVID-19 Diagnosis", ACS Central Science, vol.6, pp.591-605, 2020

[2]. Wang, Y., Wang, Y., Chen, Y., and Qin, Q. (2020) Unique Epidemiological and Clinical Features of the Emerging 2019 Novel Coronavirus Pneumonia (COVID-19) Implicate Special Control Measures. J. Med. Virol. 92, 568-576

[3]. Wang, C., Horby, P. W., Cao, B., Wu, P., Yang, s., Gao, H., Li, H., Tsang, T. K., Liao, Q., et al. (2014) Comparison of Patients Hospitalized with Influenza A Subtypes H7N9, H5N1, and 2009 Pandemic H1N1. Clin. Infect. Dis. 58 (58), 1095-1103.

[4]. Islam MM, Hannan T, Sarker L, Ahmed Z. COVID-DenseNet: a deep learning architecture to detect COVID-19 from chest radiology images. https://doi.org/ 10.20944/preprints202005.0151.v1, 2020.

[5] Ng M-Y, Lee EY, Yang J, Yang F, Li X, Wang H, et al. Imaging profile of the COVID-19 infection: radiologic findings and literature review. Radiology: Cardiothor Imag 2020;2(1).

[6]. M. Srivastava, N. Srivastava, P.K. Mishra, B.D. Malhotra "Prospects of nanomaterials-enabled biosensors for COVID-19 detection", Science of the Total Environment, 754(2021), pp. 1-12, 2020.

[7]. O.S. Albahri, A.A. Zaidana, A.S. Albahri, B.B. Zaidana, K. H. Abdulkareemc, Z.T. Al-qaysi ,A.H. Alamoodi ,A.M. Aleesa, M.A. Chyada, R.M. Alesa , L.C. Kema, M. Modi Lakulua, A.B. Ibrahima, N. A. Rashid "Systematic review of artificial intelligence techniques in the detection and classification of COVID-19 medical images in terms of evaluation and benchmarking: Taxonomy analysis, challenges, future solutions and methodological aspects", Journal of Infection and Public Health, 13(2020) pp.1381-1396, 2020.

[8]. N. N. Das, N. Kumar, M. Kaur, V. Kumar, D. Singh “Automated Deep Transfer Learning-Based Approach for Detection of COVID-19 Infection in Chest X-rays", IRBM, 2020, https://doi.org/10.1016/j.irbm.2020.07.001.

[9]. X. Yuan, C. Yang, Q. He, J. Chen, D. Yu, J. Li, S. Zhai, Z. Qin, K. Du, Z. Chu, P. Qin "Current and Perspective Diagnostic Techniques for COVID-19", ACS Infectious Disease, vol.6, issue.8 pp.1998-2016, 2020.

[10] C. H. Chau, J. D. Strope, W. D. Figg "COVID-19 Clinical Diagnostics and Testing Technology", Pharmacotherapy: The Journal of Human Pharmacology and Drug Therapy Volume 40, Issue 8 